\title{
Characterization of mercerized cellulose nanofibrils prepared by aqueous counter collision process
}

\author{
Daisuke Tatsumi, Atsushi Kanda and Tetsuo Kondo * (i)
}

\begin{abstract}
Cellulose nanofibrils (CNFs) obtained by aqueous counter collision (ACC) methods have amphiphilic Janus-type properties, which appear markedly for ACC-CNFs prepared from bacterial nanocellulose (BNC) pellicles. The amphiphilic Janus-type surface is exposed because of the mechanism involved in ACC pulverizing of cellulose materials, in which the predominant interactions of the $(200)$ lattice plane of the cellulose I crystal structure are weak interplanar van der Waals interactions. Such selective cleavage is more likely to occur for highly crystalline BNC. This study focused on alkali-mercerized cellulose samples, which are of lower crystallinity than BNC. The mercerized raw materials were subjected to ACC treatments and their fiber morphologies, crystallinities, and surface properties were compared to those of ACC-CNFs from native samples. In particular, the Wide-angle X-ray diffraction (WAXD) results suggested that the cleavage was most likely to occur at the (1 110$)$ plane in nanofibrils derived from cellulose Il, unlike (2 0 0) lattice plane for the case of cellulose I. Accordingly, the entire results indicate that the properties of the ACC-treated mercerized CNFs differ greatly from those of conventional ACC-CNFs composed of cellulose I crystalline structure. This is presumably because ACC nanopulverization proceeds depending on the surface structure and crystalline morphology of the raw material.
\end{abstract}

Keywords: Mercerized cellulose, Aqueous counter collision, Cellulose nanofibril, Cellulose Il crystal, Nanopulverization

\section{Introduction}

Cellulose nanofibrils (CNFs) are attracting increasing interest for use as building blocks of high-performance materials obtained from renewable sources. They are now widely used as fillers for composite materials [1-8] because of their excellent mechanical properties.

CNFs are generally obtained by mechanical or physicochemical pulverization via fibrillation of natural cellulose pulp fibers to nanometer-scale widths. Three types of processes are typically used to fabricate CNFs [8]. The first two are using mechanical shearing stress with chemical pretreatments, e.g., by TEMPO oxidization [9],

\footnotetext{
*Correspondence: tekondo@agr.kyushu-u.ac.jp

Graduate School of Bioresource and Bioenvironmental Sciences, Kyushu University, Motooka 744, Nishi-ku, Fukuoka 819-0395, Japan
}

and solely mechanical processes, such as grinding [10]. The third type, i.e., the aqueous counter collision (ACC) method, is a physicochemical process, which involves impingement of two high-speed jets of aqueous suspensions of the raw materials, which are expelled through a pair of opposing nozzles [11-13].

The key aspect of this physicochemical process for the preparation of CNFs is selective interfacial cleavage of weak intermolecular interactions by a propagating elastic-plastic wave generated by the impingement of water jets. The shock waves resulting from ACCs are propagated through the elastic crystalline domains of the initial materials, and thus cleave between the hydrophobic van der Waals $\left(\begin{array}{lll}2 & 0 & 0\end{array}\right)$ planes normally hidden inside native crystalline cellulose fibers. These hydrophobic planes are eventually exposed on the
Springer Open
C The Author(s) 2022. Open Access This article is licensed under a Creative Commons Attribution 4.0 International License, which permits use, sharing, adaptation, distribution and reproduction in any medium or format, as long as you give appropriate credit to the original author(s) and the source, provide a link to the Creative Commons licence, and indicate if changes were made. The images or other third party material in this article are included in the article's Creative Commons licence, unless indicated otherwise in a credit line to the material. If material is not included in the article's Creative Commons licence and your intended use is not permitted by statutory regulation or exceeds the permitted use, you will need to obtain permission directly from the copyright holder. To view a copy of this licence, visit http://creativecommons.org/licenses/by/4.0/. 
surface [14]. The resulting CNFs (ACC-CNFs) were found to have a Janus-type amphiphilic surface that is composed of both hydrophobic and hydrophilic planes in an aqueous system [15]. In addition, ACC-CNFs reflect the characteristics of the raw material [16].

Natural cellulose fibers have an internal structure in which the reducing ends of cellulose molecules are aligned in parallel and in one direction. When natural cellulose fibers are subjected to ACC treatment, the ACC-CNFs are expected to be refined from the reducing end during the preparation process. This is confirmed by the observation that thin fibrillated fibers were fibrillated at one end of the thick raw fibers [13, 17].

Mercerization is a process in which natural cellulose fibers are swollen by immersion in highly concentrated aqueous $\mathrm{NaOH}$ solution, washed with water, and dried. During this process, the crystal structure is transformed from cellulose I to cellulose II, while retaining its solid phase. Cellulose II crystals consist of anti-parallel arrangements of cellulose molecular chains with alternating reducing ends. In the crystal, hydrogen bonding of the molecular chains occurs not only via $\mathrm{O}(2)-\mathrm{O}\left(6^{\prime}\right)$ bonds, but also via $\mathrm{O}(2)-\mathrm{O}\left(2^{\prime}\right)$ and $\mathrm{O}(6)-\mathrm{O}\left(6^{\prime}\right)$ glucose residue bonds, as shown in Fig. 1. In addition, the crystallinity lower than that of natural cellulose, and is commonly of the order of $60 \%$.

Research to date has only focused on ACC treatment of native cellulose materials. In the ACC treatment of cellulose II, it is still unclear what the surface properties are and which crystal planes are cleaved. The ACC-CNFs from cellulose II are expected to show specific properties that differ from those of conventional ACC-CNFs. In this study, commercially available microcrystalline cellulose (MCC) powder, which had been derived from wood pulp by acid hydrolysis, and its mercerized cellulose were subjected to ACC treatments. The fiber morphologies, crystallinities, and surface properties of the ACC-treated products were investigated. The results are discussed in terms of the cleavage mechanism during the ACC treatment.

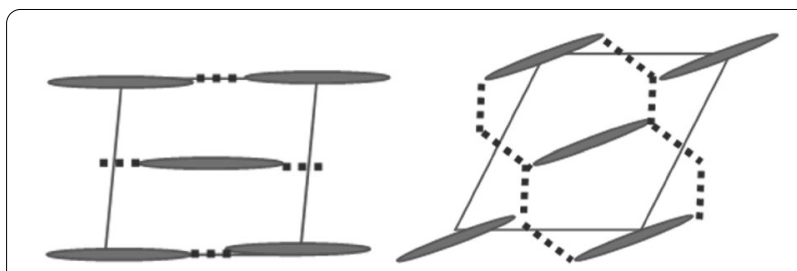

Fig. 1 Schematic diagrams of crystal lattice and hydrogen bonds of cellulose I (left) and cellulose II (right). Bars indicate glucan chains, and dotted lines indicate hydrogen bonds
It should be noted that because the samples used had similar degrees of crystallinity (ca. 60\%), it is preferable to compare the changes in their properties.

\section{Materials and methods \\ Sample preparation}

Commercially available MCC (Funacel II, Funakoshi Co., Ltd., Tokyo Japan) was used as the raw material. The degree of polymerization was 200-220 [13]. The MCC was washed three times with deionized water, twice with a 1:1 (v/v) mixture of deionized water and acetone, and twice with acetone, as described in a previous report [18]. The MCC was used as received (untreated, U). A mercerized (M) sample was obtained by immersion of the MCC in $17.5 \mathrm{wt} \% \mathrm{NaOH}$ for $30 \mathrm{~min}$, neutralizing, and washing with methanol and ethanol. The two samples were suspended in deionized water at a concentration of $1.0 \mathrm{wt} \%$, and the sample suspensions were subjected to ACC treatment at ejecting pressures of 100 and $200 \mathrm{MPa}$, and numbers of collisions from 5 to 90 Pass.

The samples were labeled as shown in Table 1; $\mathrm{U}$ and $\mathrm{M}$ indicate un-mercerized and mercerized, respectively; 100 or 200 is the ejecting pressure and the last numbers are the Pass numbers.

\section{Measurement of fiber lengths and widths}

One of the obtained sample dispersions, M200-60, was diluted to $1.0 \times 10^{-4} \mathrm{wt} \%$, cast on a glass substrate, airdried for more than 1 day, and then examined by atomic force microscopy (AMF). All images were obtained using an MFP-3D Origin instrument (Oxford Instruments Asylum Research, Inc., Santa Barbara, CA, USA), which was operated in AC mode, with a Si-cantilever (OMCLAC240TS, Olympus Co., Tokyo, Japan), at room temperature. All the obtained images were processed using the accompanying software from Oxford Instruments Asylum Research. ImageJ software was used to determine the fiber lengths from the images.

Small-angle X-ray scattering (SAXS) was performed at the SAGA Light Source BL-11 beamline (Tosu, Saga, Japan), at an energy of $8 \mathrm{keV}$ and a camera length of $2.558 \mathrm{~m}$. The scattering intensities, $I(q)$, of the sample dispersions at 60 and 90 Pass were collected using a PILATUS $300 \mathrm{k}$ detector. The data were circular-averaged using fit2d, for cross-sectional Guinier plotting [19]. The

Table 1 Pretreatments and conditions for ACC processes

\begin{tabular}{llll}
\hline Pre-treatment & $\begin{array}{l}\text { Ejecting } \\
\text { pressure/ } \\
\text { MPa }\end{array}$ & Pass number/Pass & Sample name \\
\hline Untreated (I) & 100,200 & $5,10,30,60,90$ & U100-5 U200-90 \\
Mercerization (II) & 100,200 & $5,10,30,60,90$ & M100-5 M200-90 \\
\hline
\end{tabular}




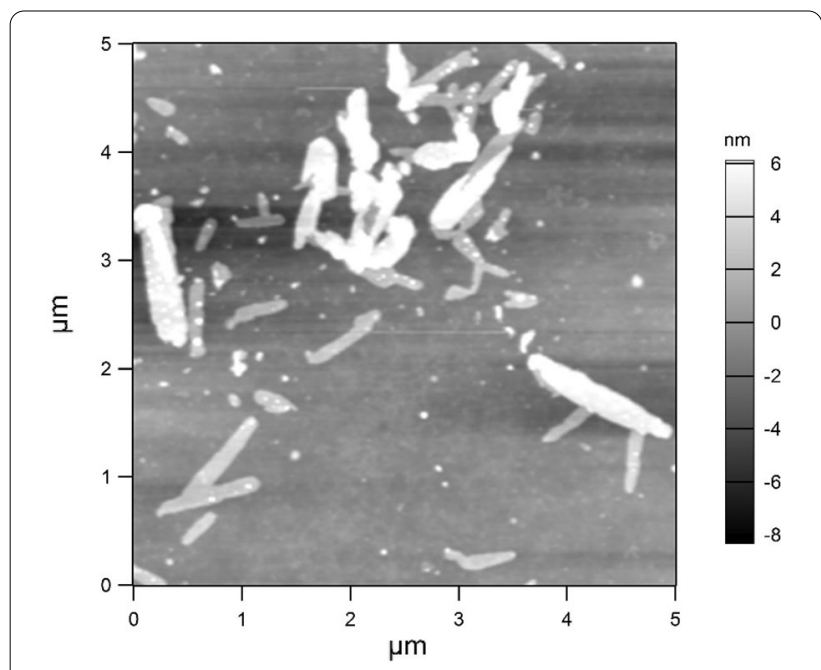

Fig. 2 AFM image of M200-60

fiber radius, $r\left(r=\sqrt{2} R_{\mathrm{c}}\right.$ ), which corresponds to the fiber width, was calculated from the cross-sectional radius of gyration, $R_{\mathrm{c}}$, from plots constructed using Eq. (1).
Table 2 Fiber width of samples calculated by cross-sectional Guinier plots

\begin{tabular}{llllll}
\hline Sample & $\boldsymbol{R}_{\mathrm{c}} / \mathrm{nm}$ & $\begin{array}{l}\text { Fiber width/ } \\
\mathbf{n m}\end{array}$ & Sample & $\boldsymbol{R}_{\mathrm{c}} \mathbf{\text { nm }}$ & Fiber width/nm \\
\hline U100-60 & 3.55 & 10.04 & M100-60 & 3.16 & 8.94 \\
U100-90 & 3.98 & 11.26 & M100-90 & 3.16 & 8.96 \\
U200-60 & 3.64 & 10.3 & M200-60 & 3.01 & 8.50 \\
U200-90 & 3.49 & 9.88 & M200-90 & 3.17 & 8.96 \\
\hline
\end{tabular}

$$
\ln I(q) q=\ln I(0) q-\frac{1}{2} R_{c}^{2} q^{2}
$$

\section{Crystallographic properties}

Wide-angle X-ray diffraction (WAXD; SmartLab, Rigaku, Inc., Tokyo, Japan) analysis of freeze-dried samples was performed in the range $2 \theta=5^{\circ}-40^{\circ}$ at a current of $30 \mathrm{~mA}$ and a voltage of $40 \mathrm{kV}$. After removing the amorphous halos, which were caused by the glass substrates, from the obtained diffraction images, the changes in the crystal structures were investigated.
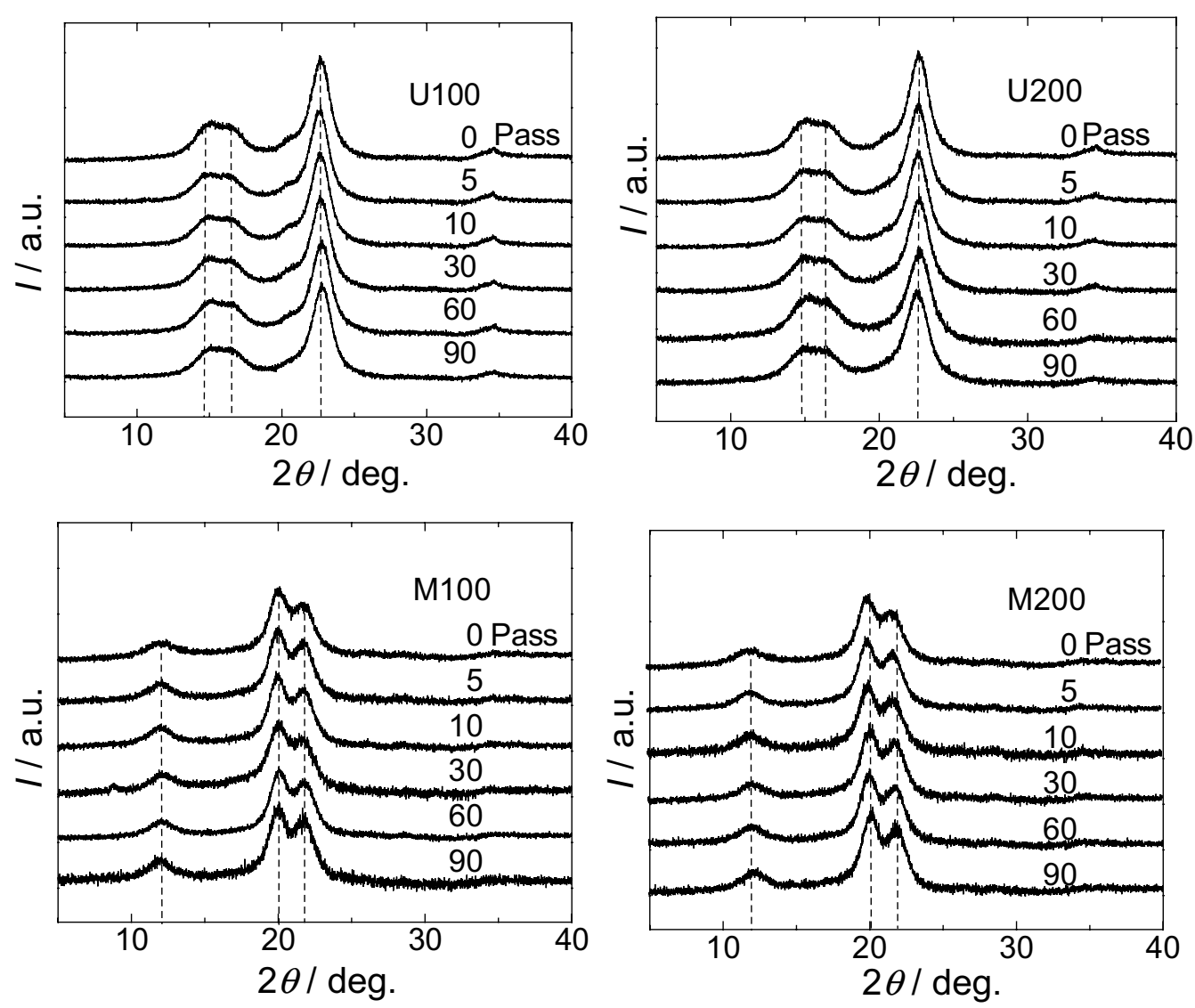

Fig. 3 WAXD profiles of ACC-CNFs from untreated $(U)$ and mercerized $(M)$ MCCs 


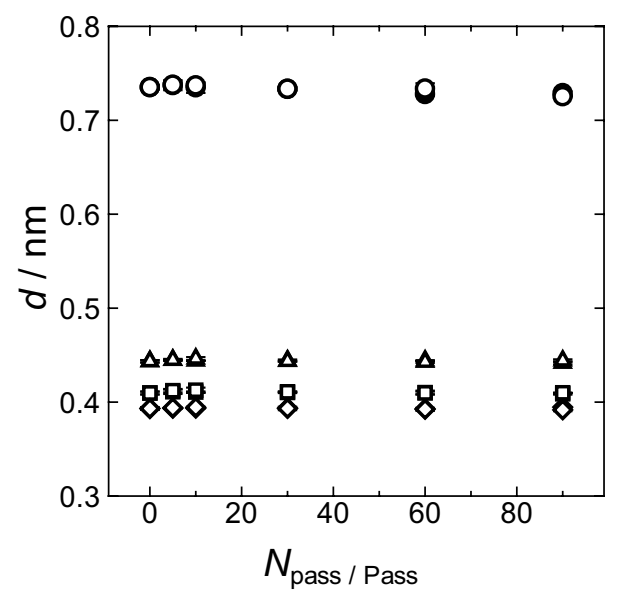

Fig. 4 Relationships between lattice spacing in products and ACC conditions. Diamonds: (2 00$)$ plane of U-series; circles: $\left(\begin{array}{lll}1 & \overline{1} & 0\end{array}\right)$ plane, triangles: $\left(\begin{array}{lll}1 & 1 & 0\end{array}\right)$ plane, and squares: $\left(\begin{array}{ll}0 & 2\end{array}\right)$ plane of M-series. Open and filled symbols indicate ACC ejecting pressures of 100 and $200 \mathrm{MPa}$, respectively law:

The lattice spacing, $d$, was determined using Bragg's

$$
2 d \sin \theta=n \lambda
$$

where $\theta$ is the $\mathrm{X}$-ray incident angle, $\lambda$ is the wavelength of the incident $\mathrm{X}$-ray, and $n$ represents the reflection order ( $n=1$ in this case).

The Scherrer equation was used to calculate the crystal width, $w[20]$ :

$$
w=\frac{0.9 \lambda}{\beta_{\frac{1}{2}} \cos \theta}
$$

where $\beta_{1 / 2}$ is the half-width of the diffraction line.

The Segal method $[21,22]$ was used to estimate the crystallinity, $C I$ :

$$
C I(\%)=\left(1-\frac{I_{\mathrm{am}}}{I_{\mathrm{cr}}}\right) \times 100
$$

where $I_{\mathrm{am}}$ and $I_{\mathrm{cr}}$ are the X-ray diffraction intensities from the amorphous and crystalline regions, respectively. $I_{\mathrm{cr}}$ is the intensity from the $(200)$ plane in cellulose I and from the $\left(\begin{array}{lll}0 & 2 & 0\end{array}\right)$ plane in cellulose II. For the lattice spacing, crystal width, and crystallinity, the WAXD measurements were repeated three times and the mean values were plotted versus the Pass number.

\section{Surface properties}

The fractal dimension, $D$, of the ACC-treated fibers was calculated from their SAXS profiles. The dependence of the intensity, $I(q)$, on the scattering vector, $q$, was introduced into $D$ by extrapolation of the approximate straight lines of the profiles.

Each sample subjected to ACC treatment at $200 \mathrm{MPa}$ and 60 Pass was adsorbed on isotactic polypropylene (iPP; H-500, Prime Polymer Co., Ltd.) and polystyrene (PS; S-20, Sekisui Chemical Industries, Ltd.) particles [16]. The diameter, density, and surface tension of the iPP particles were $500 \mu \mathrm{m}, 0.9 \mathrm{~g} / \mathrm{cm}^{3}$, and $29 \mathrm{mN} / \mathrm{m}$, respectively [23]. The diameter, density, and surface tension of the PS particles were $300 \mu \mathrm{m}, 1.05 \mathrm{~g} / \mathrm{cm}^{3}$, and $40 \mathrm{mN} / \mathrm{m}$, respectively [23].

The added amounts of polymer particles and suspension were calculated according to previous reports [16, 24]. The mixed liquid was stirred at $150 \mathrm{rpm}$ for $60 \mathrm{~min}$ at room temperature to sufficiently adsorb the nanofibrils on the particles. Excess nanofibrils were removed by washing with deionized water and then the particles were dried at $50{ }^{\circ} \mathrm{C}$ for 1 day or more. The polymer particles were stained with calcofluor white (CFW), which is a fluorescent probe specific for cellulose. Excess CFW was removed by washing with deionized water, and the particles were dried by the procedure used for drying the unstained particles. The adsorption of the fibers on each type of polymer particle was examined by confocal laser scanning microscopy (CLSM; TCS SP8 STED, Leica Inc., Wetzlar, Germany) at $410-470 \mathrm{~nm}$, with an excitation wavelength of $405 \mathrm{~nm}[15,16]$.

\section{Results and discussion} Fiber length and width

An AFM image of M200-60 is shown in Fig. 2. The fibers of the ACC-treated mercerized MCCs were shorter than those of ACC-CNFs derived from BNC [15]. Image $\mathrm{J}$ software was used to estimate the mean fiber length, which was $773.7 \pm 343.8 \mathrm{~nm}(n=65)$, and the mean fiber width (as height), which was $5.57 \pm 2.06 \mathrm{~nm}$ $(n=50)$. AFM enabled determination of the fiber lengths and widths under dry conditions. Cross-sectional plots obtained from SAXS profiles were used to determine the widths of the fibers in suspension. The data are shown in Table 2. The data obtained using AFM and SAXS show that the widths of cellulose II-derived fibers tend to be smaller than those of cellulose I-derived ones. These results indicate that the degree of pulverization may differ depending on the crystal structure of the raw material, i.e., cellulose I or II.

\section{Crystallographic properties}

The WAXD profiles of the samples confirmed that the U-series samples had the crystal structure of cellulose I, whereas the M-series samples had the crystal structure of cellulose II. This shows that both series of samples 

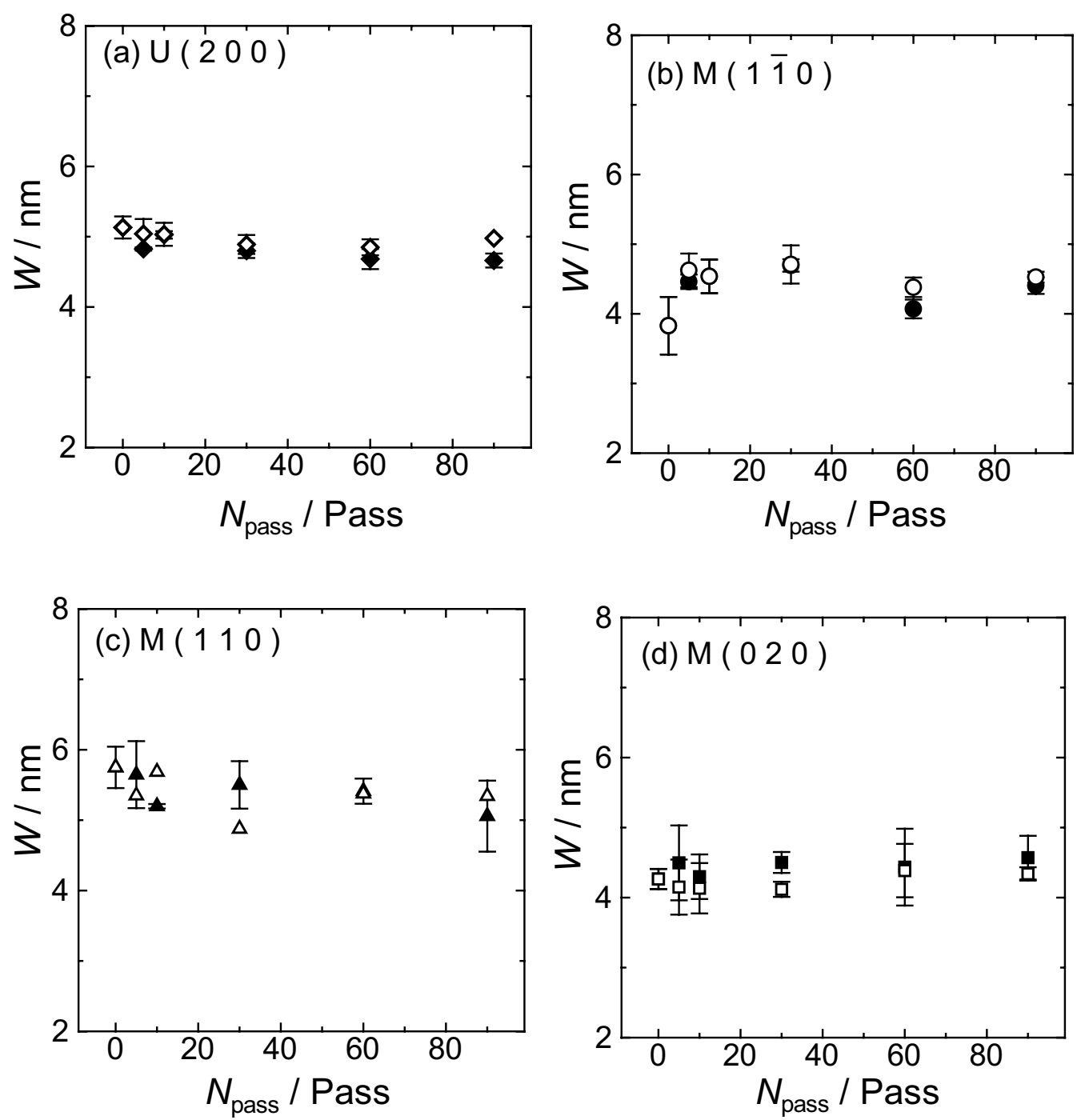

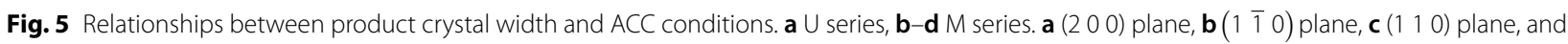
d $(02$ 0) plane. Open and filled symbols indicate ACC ejecting pressures of 100 and 200 MPa, respectively

retained the original crystal structure after ACC treatment (Fig. 3), although different collision pressures and Pass numbers were used. The diffraction peak from the (2 00 ) plane is the most intense for cellulose I, at which the ACC cleavage is considered to occur. The individual lattice spacings for the ( $\left.\begin{array}{lll}1 & \overline{1} & 0\end{array}\right),\left(\begin{array}{lll}1 & 1 & 0\end{array}\right)$, and $\left(\begin{array}{lll}0 & 2 & 0\end{array}\right)$ planes of cellulose II were calculated using Eq. (2). The relationship between the lattice spacings and ACC conditions is shown in Fig. 4; the open symbols (ACC at $100 \mathrm{MPa}$ ) and the filled symbols (ACC at $200 \mathrm{MPa}$ ) are nearly superimposed. Each plot shows that the lattice spacings are almost constant, i.e., they are independent of the Pass number. A previous study showed that the lattice spacing and crystal width are almost constant, regardless of the number of collisions, for cellulose I under an ejecting pressure of $200 \mathrm{MPa}$ [13]. These results show that ACC treatment does not change the lattice spacings of mercerized cellulose I, as well as cellulose I samples, under the ejecting pressures and Pass numbers used.

The Scherrer equation was used to calculate the crystal

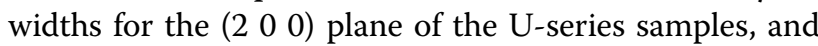
those for the ( $\left.\begin{array}{lll}1 & \overline{1} & 0\end{array}\right),\left(\begin{array}{lll}1 & 1 & 0\end{array}\right)$, and $\left(\begin{array}{lll}0 & 2 & 0\end{array}\right)$ planes for the $\mathrm{M}$-series samples. The widths before and after ACC treatment were compared, as shown in Fig. 5. The crystal width along the $\left(\begin{array}{lll}2 & 0 & 0\end{array}\right)$ plane of the U-series samples decreased with increasing Pass number. In contrast, the crystal widths along the $\left(\begin{array}{lll}1 & 1 & 0\end{array}\right)$ and $\left(\begin{array}{ll}0 & 2\end{array}\right)$ planes of the M-series samples did not depend on the Pass number. However, the 

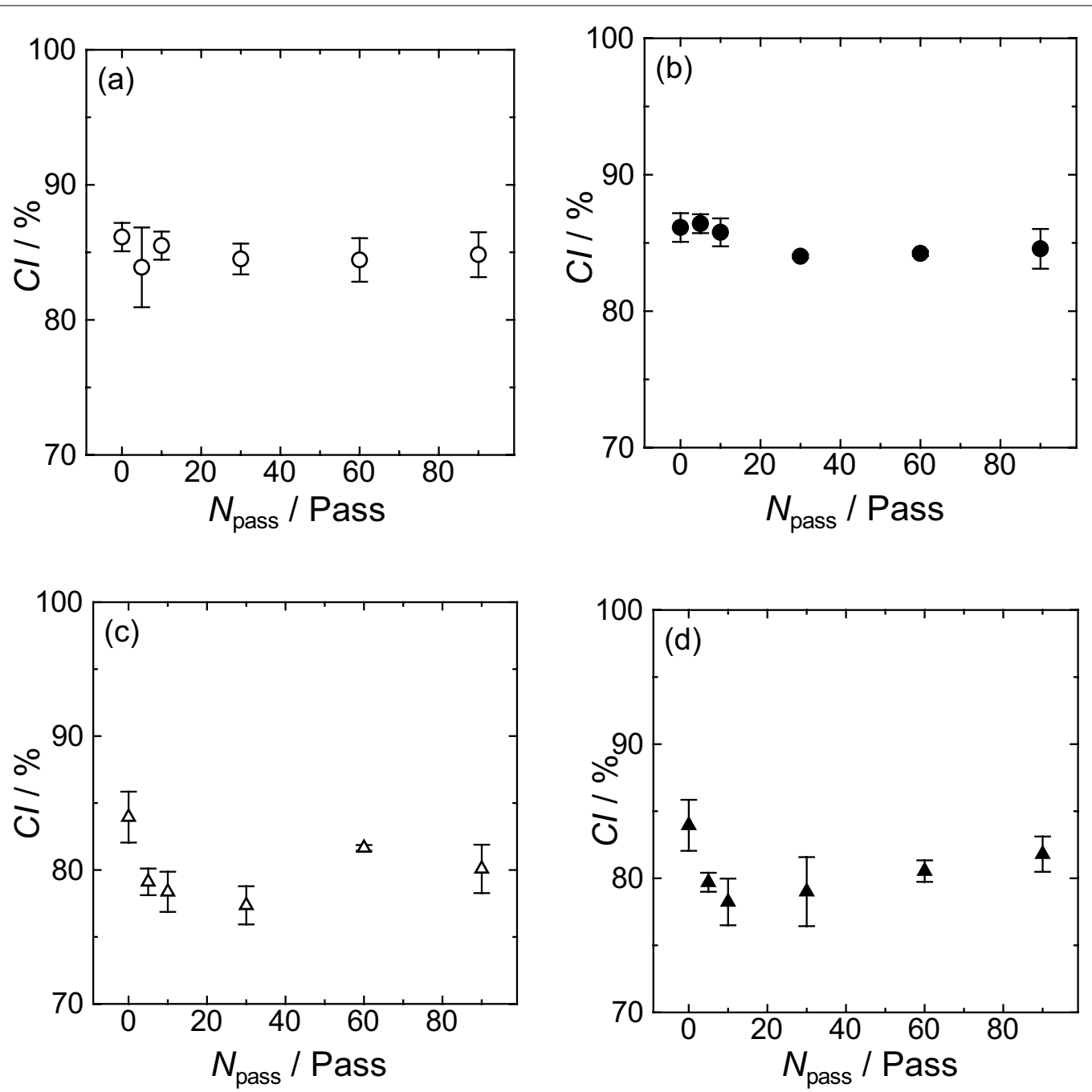

Fig. 6 Relationships between product crystallinity and ACC conditions: a U100, b U200, c M100, and d M200

crystal width along the (1 110$)$ plane tended to decrease with increasing Pass number. The decrease in the crystal width along the $\left(\begin{array}{lll}2 & 0 & 0\end{array}\right)$ plane of the U-series sample indicates that cleavage during ACC treatment probably occurs at this plane. In contrast, the crystal width along the (1 110$)$ plane tended to decrease with increasing Pass number for the $\mathrm{M}$-series samples. The value obtained by dividing $W$ by each $d$-spacing $(W / d)$ indicates how many layers of crystal width decreased in $d$-spacing units. The value exhibited that the widths for $\mathrm{U}$ - and $\mathrm{M}$-series samples decreased by about 10 layers along the $\left(\begin{array}{lll}2 & 0 & 0\end{array}\right)$ and ( $\left.\begin{array}{lll}1 & 1 & 0\end{array}\right)$ planes, respectively. It is, therefore, assumed that cleavage during ACC treatment begins at the $\left(\begin{array}{lll}1 & 1 & 0\end{array}\right)$ plane in mercerized cellulose. However, a more detailed investigation is needed. The changes in the crystallinity of each sample are shown in Fig. 6. According to a previous report [13], when cellulose I fibers were subjected to ACC treatment at $200 \mathrm{MPa}$, the crystallinity initially decreased with increasing Pass number and then increased. In the current study, similar behavior was observed during ACC treatment of cellulose II.

\section{Surface properties}

Figure 7 shows double-logarithmic plots of the SAXS peak intensities versus the scattering vector, $q$. The relationship in the $q$ range from -1 to 0 is linear for U100 and convex upward for U200. For both M-100 and 

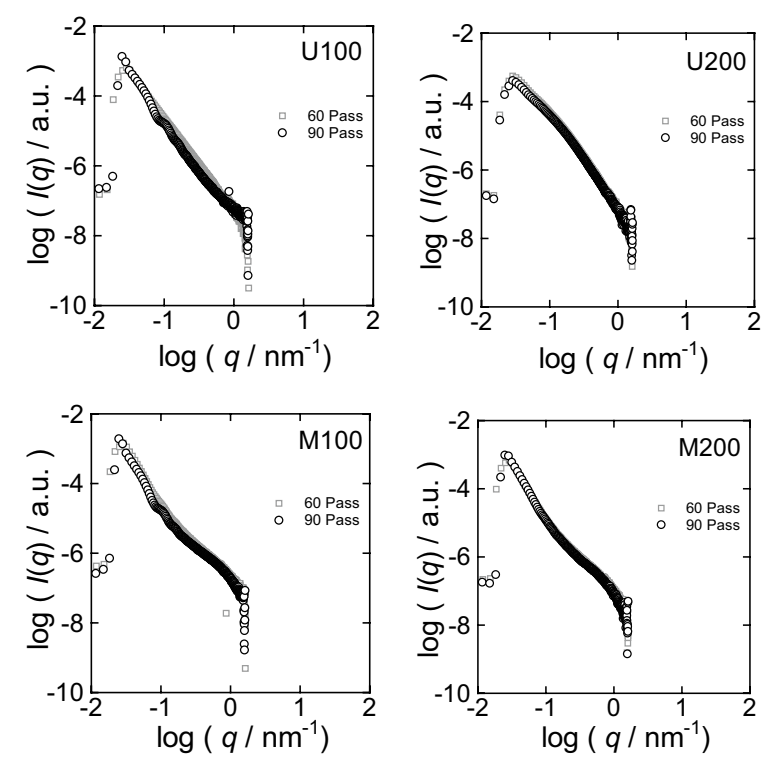

Fig. 7 Dependences of intensities, $I(q)$, on scattering vector, $q$

Table 3 Fractal dimensions, D, obtained from SAXS profiles

\begin{tabular}{llll}
\hline Sample & D & Sample & D \\
\hline U100-60 & 2.69 & M100-60 & 1.77 \\
U100-90 & 2.40 & M100-90 & 1.60 \\
U100-60 & 2.96 & M100-60 & 1.82 \\
U100-90 & 2.95 & M100-90 & 1.83 \\
\hline
\end{tabular}

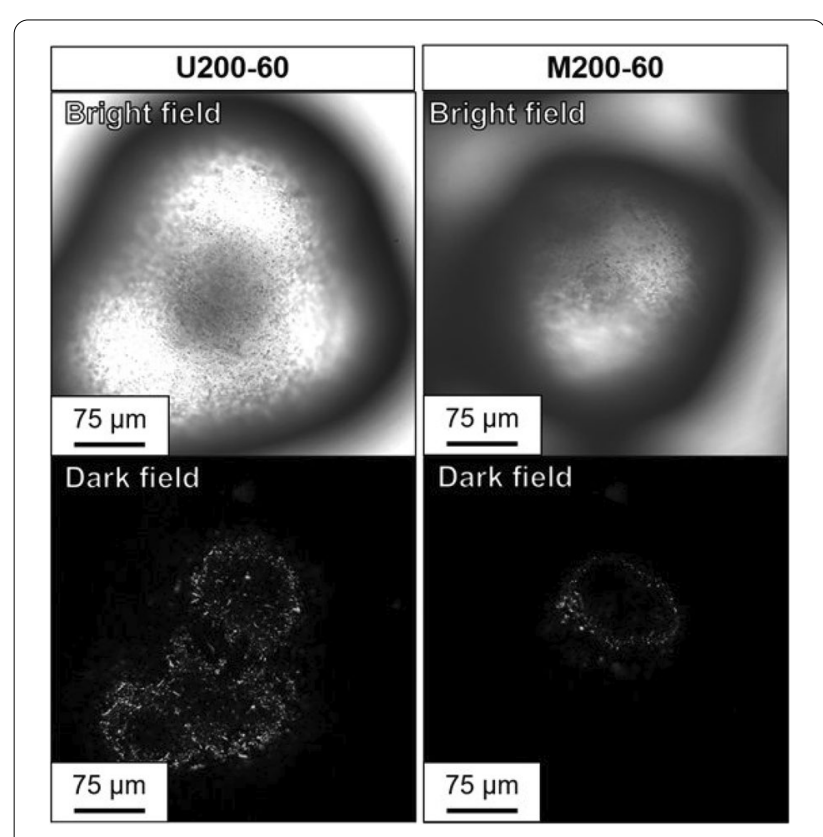

Fig. 8 CLSM images of iPP particles with adsorbed nanofibrils

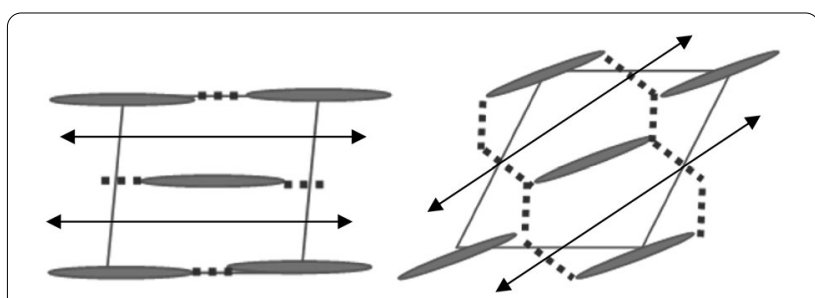

Fig. 9 Schematic diagrams of expected cleavage plane in the crystal lattice for cellulose I (left) and cellulose II (right). Double-headed arrows indicate expected cleavage planes

M-200, the relationship is convex downward. The fractal dimension, $D$, which was calculated from the approximate straight lines in the U-series plots, is close to 2.5 for $\mathrm{U} 100$ and 3 for U200. This shows that $D$ increases with increasing degree of nano-pulverization. In contrast, no differences between the $D$ values for cellulose II were observed at the different ejection pressures, i.e., both are below 2 (Table 3 ). The fractal dimensions, $D$, obtained from the SAXS profiles reflect the presence of structures with dimensions of the order of several to $100 \mathrm{~nm}$ in the samples [25]. It should be noted that a structure is twodimensional if $D$ is close to 2 , but three-dimensional if it is close to 3. The surfaces of the M-series samples are, therefore, assumed to be more planar than those of the U-series samples. It has been reported that ACC-CNFs are formed by progressive nano-pulverization during fibrillation [13]. It is possible that the increase in the fractal dimension caused by the ACC ejecting pressure as nano-refinement progresses reflects formation of finer nanofibrils on the surface. Before ACC treatment, the mercerized cellulose starting material has a smooth surface and a uniform internal structure [26]. It is expected that a planar fractal dimension will be observed after ACC treatment. This implies that during pulverization mercerized cellulose maintains its homogeneous internal architecture under the same ACC-treatment conditions as those used for formation of native ACC-CNFs.

Finally, the surface properties of the ACC products were investigated by examining their adsorption on polymer particles $[15,16]$. Figure 8 shows CLSM images of polymer particles coated with the produced ACCCNFs. Fluorescence attributed to CFW on the CNFs is observed in the iPP particle images. CFW stains cellulose; therefore, these images indicate that nanofibrils of cellulose I and cellulose II are both adsorbed on the iPP particle surfaces. However, the amount of cellulose II adsorbed was less than that of cellulose I. In addition, neither type of nanofibril was adsorbed on PS particles. The results suggest that the capacity for ACC-mercerized 
CNF adsorption on the iPP particle surfaces was lower than that for cellulose I-derived ACC-CNFs. This indicates that the differences among the surface properties of the fiber samples can be attributed to differences in the surfaces exposed by ACC cleavage. The adsorption of ACC-CNFs from cellulose I on iPP particles is, therefore, verified to be the result of exposure of the van der Waals surface, i.e., the (2 00$)$ plane, by the ACC treatment [16].

The WAXD results suggest that cleavage occurred at the $\left(\begin{array}{lll}1 & 1 & 0\end{array}\right)$ plane in nanofibrils derived from cellulose II. Yamane et al. used molecular mechanics simulations to investigate the surface properties of cellulose II. They reported that the $\left(\begin{array}{lll}1 & \overline{1} & 0\end{array}\right)$ plane is hydrophilic, and the (1 $10)$ plane is more hydrophobic, in cellulose II [27]. During ACC treatment of cellulose II, the hydrophobic (1 1 0 ) plane is probably exposed, as in the case of cellulose I. However, in the case of cellulose II, hydrogen bonds are also present between molecular chain sheets; therefore, this plane is probably less hydrophobic than the $\left(\begin{array}{lll}2 & 0 & 0\end{array}\right)$ plane of cellulose I.

\section{Conclusion}

The behavior of mercerized CNFs obtained by ACC treatment of mercerized cellulose indicates that the surface structure of the mercerized CNFs depends on the crystal structure of the raw material, similarly to the case for conventional native CNFs. The crystal structure of mercerized cellulose differs from that of native cellulose; therefore, it is indicated that the lattice plane at which cleavage begins during ACC treatment also differs. The most probable cleavage plane in cellulose II is the $\left(\begin{array}{ll}1 & 1\end{array}\right.$ $0)$ plane as shown in Fig. 9. This will be clarified in the future using methods, such as double staining [15].

These findings suggest that a surface with various characteristics can be exposed on the CNFs by changing the crystal structures of the original cellulose and subjecting the original material to ACC treatment. This will enable the development of non-chemical surface-modification methods.

\section{Abbreviations}

ACC: Aqueous counter collision; AFM: Atomic force microscopy; BNC: Bacterial nanocellulose; CFW: Calcofluor white; Cl: Crystallinity index; CLSM: Confocal laser scanning microscopy; CNFs: Cellulose nanofibrils; iPP: Isotactic polypropylene; MCC: Microcrystalline cellulose; PS: Polystyrene; SAXS: Small-angle $X$-ray scattering; WAXD: Wide-angle X-ray diffraction.

\section{Acknowledgements}

We thank Helen McPherson, PhD from Edanz for editing a draft of this manuscript.

\section{Authors' contributions}

DT: Research plan and mainly responsible for preparation of the paper. AK: Mainly responsible for performing all experiments. TK: Research plan through study and preparation of the paper.
Funding

Not applicable.

Availability of data and materials

Not applicable.

\section{Declarations}

\section{Competing interests}

The authors declare that they have no competing interests.

Received: 23 November 2021 Accepted: 14 February 2022

Published online: 03 March 2022

\section{References}

1. Wang B, Sain M (2007) Isolation of nanofibers from soybean source and their reinforcing capability on synthetic polymers. Compos Sci Technol 67:2521-2517

2. Fujisawa S, Saito T, Kimura S, Iwata T, Isogai A (2013) Surface engineering of ultrafine cellulose nanofibrils toward polymer nanocomposite materials. Biomacromol 14:1541-1546

3. Annamalai PK, Dangon KL, Monemian S, Foster EJ, Rowan SJ, Weder C (2014) Water-responsive mechanically adaptive nanocomposites based on styrene-butadiene rubber and cellulose nanocrystals-processing matters. ACS Appl Mater Interfaces 6:967-976

4. Iwamoto S, Yamamoto S, Lee SH, Endo T (2014) Solid-state shear pulverization as effective treatment for dispersing lignocellulose nanofibers in polypropylene composites. Cellulose 21:1537-1580

5. Sato A, Kabusaki D, Okumura H, Nakatani T, Nakatsubo F, Yano H (2016) Surface modification of cellulose nanofibers with alkenyl succinic anhydride for high-density polyethylene reinforcement. Compos A 83:72-79

6. Wang L, Okada K, Hikima Y, Ohshima M, Sekiguchi T, Yano H (2019) Effect of cellulose nanofiber (CNF) surface treatment on cellular structures and mechanical properties of polypropylene/CNF nanocomposite foams via core-back foam injection molding. Polymers 11:00249

7. Kargarzadeh H, Mariano M, Huang J, Lin N, Ahmad I, Dufresne A, Thomas S (2017) Recent developments on nanocellulose reinforced polymer nanocomposites: a review. Polymer 132:368-393

8. Kondo T. Cellulose nanofibrils pulverized from biomass resources: past, present, and future perspectives. KONA Powder Part J (in press) (2022)

9. Isogai A, Saito T, Fukuzumi H (2011) TEMPO-oxidized cellulose nanofibers. Nanoscale 3:71-85

10. Abe K, Iwamoto S, Yano H (2007) Obtaining cellulose nanofibers with a uniform width of $15 \mathrm{~nm}$ from wood. Biomacromol 8:3275-3278

11. Kondo T, Morita, M, Hayakawa, K, Onda Y (2008) Wet pulverizing of polysaccharides. U.S. Patent 7,357,339

12. Kose R, Mitani I, Kasai W, Kondo T (2011) "Nanocellulose" as a single nanofiber prepared from pellicle secreted by Gluconacetobacter xylinus using aqueous counter collision. Biomacromol 12:716-720

13. Kondo T, Kose R, Naito H, Kasai W (2014) Aqueous counter collision using paired water jets as a novel means of preparing bio-nanofibers. Carbohydr Polym 112:284-290

14. Li Q, Renneckar S (2011) Supermolecular structure characterization of molecularly thin cellulose I nanoparticles. Biomacromol 12:650-659

15. Tsuji T, Tsuboi K, Yokota S, Tagawa S, Kondo T (2021) Characterization of an amphiphilic Janus-type surface in the cellulose nanofibril prepared by aqueous counter collision. Biomacromol 22:620-628

16. Ishikawa G, Tsuji T, Tagawa S, Kondo T (2021) Adsorption of Janus-type amphiphilic cellulose nanofibrils onto microspheres of semi-crystalline polymers. Macromolecules 54:9393-9400

17. Utsunomiya $H$, Tsujita Y, Kondo T (2021) Cellulose nanoanemone: an asymmetric form of nanocellulose. Cellulose. https://doi.org/10.1007/ s10570-021-04231-9

18. Huan S, Yokota S, Bai L, Ago M, Borghei M, Kondo T, Rojas OJ (2017) Formulation and composition effects in phase transitions of emulsions costabilized by cellulose nanofibrils and an ionic surfactant. Biomacromol 12:4393-4404

19. Kratky O, Glatter O (1982) Small-angle X-ray scattering. Academic Press 
20. Shcerrer P (1918) Bestimmung der Größe und der inneren Struktur vonKolloidteilchen mittels Röntgenstrahlen. Nachrichten von der Gesellschaft der Wissenschaften zu Göttingen 26:98-100

21. Segal L, Creely JJ, Martin AE, Conrad CM (1959) An empirical method forestimating the degree of crystallinity of native cellulose using X-ray diffractometer. Text Res J 29:786-794

22. Kamide K, Okajima K, Matsui T, Kowsaka K (1984) Study on the solubility of cellulose in aqueous alkali solution by deuteration IR and ${ }^{13} \mathrm{C}$ NMR. Polym J 116:857-866

23. Brandrup J, Immergut EH (1989) Polymer handbook, pp VI411-426. New York: Wiley. ibid (1975) p II 221

24. Ishikawa G, Kondo T (2017) Thermodynamic effect on interaction between crystalline phases in size-controlled ACC-bacterial nanocellulose and poly(vinyl alcohol). Cellulose 24:5495-5503

25. Ishii D, Tatsumi D, Matsumoto T (2003) Effect of solvent exchange on the solid structure and dissolution behavior of cellulose. Biomacromol 4:1238-1243

26. Kim N, Imai T, Wada M, Sugiyama J (2006) Molecular directionality in cellulose polymorphs. Biomacromol 7:274-280

27. Yamane C, Aoyagi T, Ago M, Sato K, Okajima K, Takahashi T (2006) Two different surface properties of regenerated cellulose due to structural anisotropy. Polym J 38:819-826

\section{Publisher's Note}

Springer Nature remains neutral with regard to jurisdictional claims in published maps and institutional affiliations.

\section{Submit your manuscript to a SpringerOpen ${ }^{\circledR}$ journal and benefit from:}

- Convenient online submission

- Rigorous peer review

- Open access: articles freely available online

- High visibility within the field

- Retaining the copyright to your article

Submit your next manuscript at $\boldsymbol{\nabla}$ springeropen.com 